positive correlation with ESR, dsDNA, C3, C4. Cytokines didn't correlate with SLEDAI.

Conclusions IL6 and TNF $\alpha$ are reliable markers of disease activity, but lack significant clinical correlation.

\section{HIERARCHICAL CLUSTER ANALYSIS OF SYSTEMIC LUPUS ERYTHEMATOSUS}

${ }^{1} \mathrm{CH}$ Suh, ${ }^{1} \mathrm{JY}$ Jung, ${ }^{2} \mathrm{HY}$ Lee, ${ }^{1} \mathrm{HA}$ Kim, ${ }^{3} \mathrm{SS} \mathrm{Kim},{ }^{4} \mathrm{~S} J$ Hong. ${ }^{1}$ Ajou University School of Medicine, Rheumatology, Suwon, Republic of Korea; ${ }^{2}$ Ajou University School of Medicine, Clinical Trial Centre, Suwon, Republic of Korea; ${ }^{3}$ Ulsan University College of Medicine, Rheumatology, Gangneung, Republic of Korea; ${ }^{4}$ Kyung Hee University Hospital, Rheumatology, Seoul, Republic of Korea

10.1136/lupus-2017-000215.235

Background and aims Systemic lupus erythematosus (SLE) is a heterogeneous disorder with diverse manifestations and serologic features. The purpose is to categorise SLE patients into similar initial characteristics.

Methods Hierarchical cluster analysis approached to 389 SLE patients and 10 laboratory values. Laboratory values were transformed into Z-score for hierarchical clustering. Ward's method as agglomeration method was a criterion applied with spearman correlation as distance metric. Clinical characteristics among clusters were examined by ANOVA with Tukey and Fisher's exact test. To find each SLE cluster using initial laboratory, linear discriminant analysis was applied.

Results Three clusters were revealed by initial laboratory data; Cluster 1 had higher anti-dsDNA antibody, ANA titer and ESR, and low complements, lymphocyte, haemoglobin and platelet counts, Cluster 2 had lower anti-dsDNA antibody, ANA titer and ESR, and Cluster 3 had lower anti-dsDNA antibody titer, WBC and lymphocyte counts, and higher ANA titer. As a result from analysing cumulative manifestations and treatment, Cluster 1 showed more frequent malar rash, alopecia and renal disease with higher SLEDAI, and more use of cyclophosphamide and azathioprine. Also, oral ulcer was developed frequently in Cluster 2. During disease duration, total and mean corticosteroids and the number of flare were higher in Cluster 1.

Conclusions With initial laboratory values, SLE patients could be divided 3 clusters. Each Cluster showed different characteristics in clinical manifestations and treatment patterns. This predictive model considered disease severity had $84.6 \%$ of total predictability.

\section{ANTI-NUCLEAR ANTIBODY PATTERN AND CLINICAL MANIFESTATION PRESENTATION IN SYSTEMIC LUPUS ERYTHEMATOSUS}

${ }^{1}$ BS Suryajaya, 'VN Moenardi, ${ }^{1}$ II Hidayat, ${ }^{2}$ L Hamijoyo. ${ }^{1}$ Faculty Of Medicine Padjadjaran University/Hasan Sadikin General Hospital, Immunology study group, Bandung, Indonesia; ${ }^{2}$ Faculty Of Medicine Padjadjaran University/Hasan Sadikin General Hospital, Immunology study group Rheumatology Division- Internal Medicine Department, Bandung, Indonesia

\subsection{6/lupus-2017-000215.236}

Background and aims Anti-nuclear antibody (ANA) pattern analysis by immunofluorescence microscopy remains an important diagnostic tools for Systemic Lupus Erythematosus (SLE) in Indonesia. Although the utilisation of ANA pattern are used by physician to diagnosed SLE, there are very sparse study to explain on SLE clinical presentation and ANA pattern in Indonesia. This study was compiled to determined ANA pattern and clinical manifestation presentation in ANA positive patients.

Methods This study was an observational cross-sectional study in Hasan Sadikin General Hospital Bandung, 217 patients that had satisfied American College of Rheumatology (ACR) Criteria (1997) and ANA pattern were positive when diagnosed with SLE. Data was acquired from "RSHS Lupus Registry" database.

Results The study population consist of 217 patients, whom 208 patients (96\%) were female and 206 patients (96\%) were Sundanese (median age was 33, ranging from age 14 to 62 years), had Speckled ANA pattern (S-ANA) 98 patients (45\%), Homogenous ANA Pattern (H-ANA) 63 patients (29\%), Nucleolar ANA Pattern (N-ANA) 23 patients (11\%), SpeckledNucleolar ANA Pattern (SN-ANA) 18 patients (8\%), and other staining patterns 15 patients (7\%).The majority of clinical manifestation in S-ANA and H-ANA patients were haematological involvement with 69 patients (70\%) and 42 patients (67\%) respectively, and N-ANA and SN-ANA were malar rash with 20 patients (87\%), and 13 patients (72\%) respectively.

Conclusions The most frequent ANA pattern among SLE patients in this study is S-ANA pattern. The most common clinical presentation found is haematological involvement in SANA and H-ANA and malar rash in N-ANA and SN-ANA.

\section{ISCHAEMIC STROKE IN SYSTEMIC LUPUS ERYTHEMATOSUS, -DISTRIBUTION OF SUBTYPES AND A RISK GENOTYPE IN THE STAT4 GENE}

${ }^{1} \mathrm{E}$ Svenungsson* ${ }^{2} \mathrm{~L}$ Hopia, ${ }^{3} \mathrm{~A}$ Laveskog, ${ }^{4} \mathrm{~A}$ Jönsen, ${ }^{5} \mathrm{D}$ Leonard ${ }^{1} \mathrm{JT}$ Gustafsson, ${ }^{1}$ I Gunnarsson, ${ }^{6} \mathrm{~A}$ Zickert, ${ }^{5} \mathrm{G}$ Nordmark, ${ }^{4} \mathrm{AA}$ Bengtsson, ${ }^{7} \mathrm{~K}$ Elvin, ${ }^{8} \mathrm{JK}$ Sandling, ${ }^{8} \mathrm{AC}$ Syvänen, ${ }^{5} \mathrm{~L}$ Rönnblom, ${ }^{2} \mathrm{M}$ Andersson. ${ }^{1}$ Karolinska Institutet, Unit of Rheumatology- Department of Medicine- Solna, Stockholm, Sweden; ${ }^{2}$ Karolinska Institutet, Department of Clinical Neuroscience, Stockholm, Sweden; ${ }^{3}$ Karolinska Institutet, Department of Neuroradiology, Stockholm, Sweden; ${ }^{4}$ Lund University, Department of Clinical SciencesSection of Rheumatology, Lund, Sweden; ${ }^{5}$ Uppsala University, Department of Medical Sciences- Section of Rheumatology, Uppsala, Sweden; ${ }^{6}$ Karolinska Institutet, Unit of Rheumatology- Department of Medicine- Huddinge, Stockholm, Sweden; ${ }^{7}$ Karolinska Institutet, Unit of Clinical Immunology- Department of Clinical Immunology and Transfusion Medicine-, Stockholm, Sweden; ${ }^{8}$ Uppsala University, Department of Medical SciencesMolecular Medicine, Uppsala, Sweden

\subsection{6/lupus-2017-000215.237}

Background and aims We investigated the distribution of ischaemic stroke subtypes, classified according the Trial of Org 10172 in Acute Stroke Treatment (TOAST) system, among patients with systemic lupus erythematosus (SLE). Genetic susceptibility in the signal transducer and activator of transcription factor 4 (STAT4) gene, defined by the single nucleotide polymorphism (SNP) rs10181656(G) were explored.

Methods We identified 69/665 SLE patients with stroke. Medical charts were retrieved and brain, cardiac and vascular imaging at the time of stroke were examined. Classification was performed according to TOAST: large-artery atherosclerosis (LAA), cardioembolism (CE), small-artery occlusion (SAO), stroke of other determined aetiology (OC) and stroke of undetermined aetiology (UE). Occurrence of the anti-phospholipid syndrome (APS) was documented. Evaluators were blinded to genotypes. General population controls $(n=658)$ and SLE patients free from previous cerebrovascular disease $(n=517)$ were used as comparators. 